\title{
CAPA LÍMITE EN LEÓN: RELACIÓN CON LOS PARÁMETROS METEOROLÓGICOS Y CON LA CONCENTRACIÓN DE CONTAMINANTES
}

\author{
Cristina Calles ${ }^{1}$, Roberto Fraile ${ }^{2}$, Ana I. Calvo ${ }^{3}$ \\ Departamento de Física, IMARENAB Universidad de León, 24071 León, España \\ ${ }^{1}$ ccalla01@estudiantes.unileon.es; ${ }^{2}$ rfral@unileon.es; ${ }^{3}$ aicalg@unileon.es
}

\section{Introducción}

La calidad del aire en una zona depende de la emisión e inmisión de los contaminantes y de los procesos que intervienen en su transporte y dispersión en la atmósfera. La Ley 34/2007 regula la calidad del aire y la protección del medio ambiente. La capa límite planetaria o PBL, se define como la capa atmosférica que interacciona directamente con la superficie terrestre, convirtiéndose en una zona de mezcla constante de los componentes atmosféricos. Juega un papel fundamental en la calidad del aire y en las condiciones meteorológicas (Selvaratnam et al., 2015). La altura de la PBL es muy variable (Seidel et al., 2012); los valores pueden oscilar desde unos pocos metros a uno o dos kilómetros. Su espesor depende de diversos factores (Núñez, 2002) como la topografía, la naturaleza de la cubierta vegetal, la velocidad del viento, las condiciones atmosféricas (clase de estabilidad), la tasa de insolación recibida, etc.

El objetivo principal de este trabajo es el estudio de la relación entre la altura de la PBL yi) algunos parámetros meteorológicos (temperatura, humedad relativa y velocidad del viento) ii) la concentración de contaminantes registrados en dos estaciones de calidad del aire de la ciudad de León, durante el año 2016.

\section{Zona de estudio}

Este estudio se ha llevado a cabo en la ciudad de León, situada al noroeste de la Península Ibérica, en la comunidad autónoma de Castilla y León. Se encuentra a una altitud de 837 metros sobre el nivel del mar. Dispone de cuatro estaciones de calidad del aire,

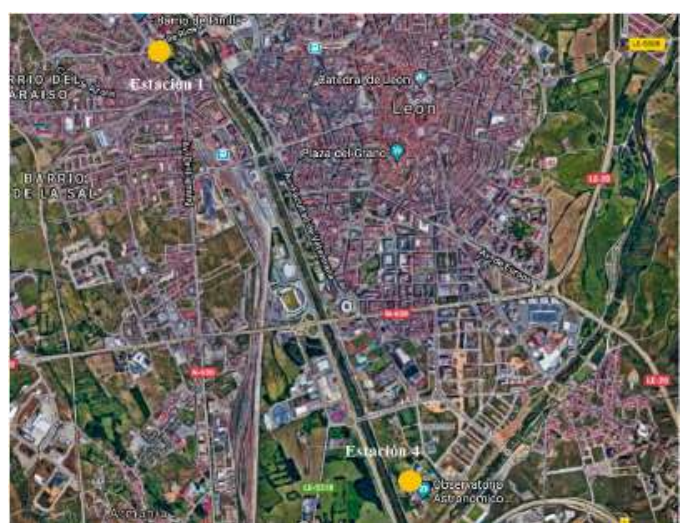
ubicadas en diferentes puntos de la ciudad, que son propiedad de la Junta de Castilla y León y que están integradas en la llamada "Red de vigilancia y control de la contaminación atmosférica en Castilla y León". Durante el año 2016, solo dos estaciones estaban operativas (León 1 y León 4) (Fig. 1).

Fig. 1.- Localización de las estaciones León 1 y León 4 


\section{Metodología}

Se crearon dos bases de datos: i) base de datos meteorológicos (a partir de radiosondeos de la NOAA, cada 3 horas) y ii) base de datos de contaminantes (a partir de la Red de Calidad del Aire de la Junta de Castilla y León). Para llevar a cabo el objetivo principal de este trabajo, se empleó el paquete estadístico SPSS (Statistical Package for the Social Sciences) para aplicar, las pruebas no paramétricas de Kruskal-Wallis y determinar el coeficiente de correlación de Pearson.

\section{Resultados}

\subsection{Análisis de la altura de la PBL}

Para el estudio estacional, se agruparon los meses como sigue: i) invierno: enero, febrero y marzo; ii) primavera: abril, mayo y junio; iii) verano: julio, agosto y septiembre y iv) otoño: octubre, noviembre y diciembre. El análisis de la evolución mensual de la capa límite planetaria, presenta una altura mayor en los meses de primavera y verano y una altura mucho menor en los meses de invierno y otoño, siendo el mes de diciembre en
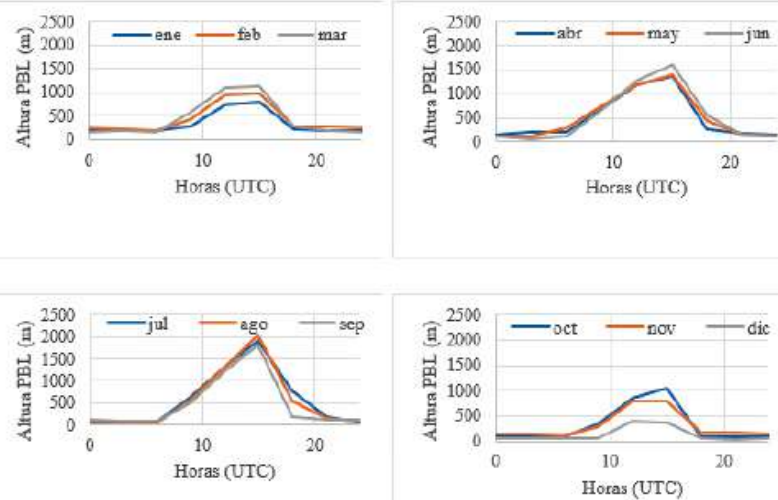
el que se observa la PBL más baja y el mes de agosto el de la más alta (Fig. 2).

Fig. 2.- Evolución horaria de la altura de la PBL a lo largo de los distintos meses de estudio.

En el gráfico de la evolución mensual de la altura de la PBL (Fig. 3) se observa un máximo para las 1500 UTC en los meses de julio, agosto y septiembre. En cambio, para las 0900 y las $1200 \mathrm{UTC}$, se observa un aumento progresivo de la altura de la PBL. Durante las horas nocturnas, la PBL se encuentra muy cerca del nivel del suelo.

\subsection{Relación entre la altura de la PBL y los parámetros meteorológicos}

Se observa un incremento de la altura de la PBL en las horas diurnas, asociado con la disminución de la humedad relativa y con un aumento de la temperatura en superficie. Esta relación entre la PBL y la temperatura y la humedad relativa ya fue descrita por Zhang et al. (2013), quienes explicaron que una temperatura superficial más alta y una humedad relativa menor dan como resultado un mayor flujo de calor sensible y un menor flujo de calor latente, lo que lleva a una convección más profunda y una altura mayor de la PBL.

$\mathrm{El}$ análisis mensual de la velocidad del viento muestra una mayor velocidad en los meses de enero a abril y menor en el resto de meses con un mínimo en diciembre. En cuanto al coeficiente de correlación de Pearson, se observa una correlación positiva entre la velocidad del viento y la altura de la PBL. 

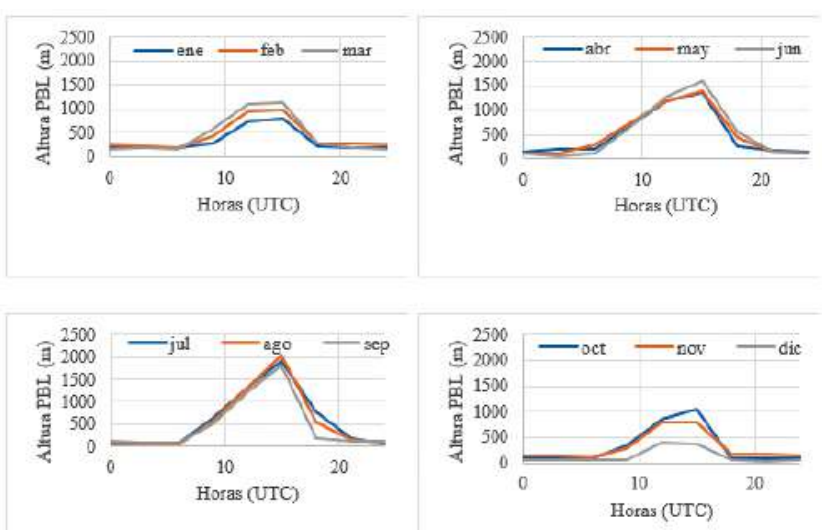

Fig. 3.- Evolución mensual de la altura de la PBL a las 0900, 1200 y 1500 UTC.

\subsection{Relación entre la altura de la PBL y la concentración de con- taminantes}

El estudio de las correlaciones entre la altura de la PBL y la concentración de contaminantes registradas en las dos estaciones de control para el año 2016 muestra que:

- En la estación León 1 (estación de tráfico), la altura de la PBL presenta una correlación negativa y significativa $(\alpha=0,01)$ con la concentración de contaminantes $\mathrm{NO}, \mathrm{NO}_{2}$, $\mathrm{PM}_{10}$ y SO$_{2}$. Una mayor altura de la PBL favorece la dispersión, por lo que la concentración de contaminantes es menor (Zou et al., 2017).

- En la estación León 4 (estación de fondo) la altura de la PBL muestra una correlación significativa $(\alpha=0,01)$ con la concentración de $\mathrm{NO}_{\text {y } \mathrm{NO}_{2}}$ (correlación negativa) y con la concentración de $\mathrm{O} 3$ (correlación positiva). Esto último puede ser debido al papel fundamental que desempeña la radiación solar en la formación de ozono troposférico. Por otro lado, se ha observado que el material particulado presenta una mayor concentración en verano, cuando la PBL tiene una mayor altura. Una posible explicación podría ser que, en verano, a pesar de que el incremento de la PBL favorece la dispersión de contaminantes, las altas temperaturas y la mayor insolación favorecen la actividad fotoquímica, provocando un aumento en la concentración de determinadas sustancias contaminantes (Calvo, 2009). Además, la contribución de otro tipo de fuentes, como las intrusiones saharianas, la quema de rastrojos o los incendios forestales, que son más frecuentes en verano, provocan una mayor concentración de contaminantes. Por otra parte, la correlación entre la altura de la $\mathrm{PBL}$ y el $\mathrm{SO}_{2}$ no resultó ser significativa.

\section{Agradecimientos}

Este trabajo ha sido parcialmente financiado por el Ministerio de Economía y Competitividad (TEC2014-57821-R), la Universidad de León (Programa Propio 2015/00054/001) y el proyecto AERORAIN (Ministerio de Economía y Competitividad, CGL2014-52556-R, cofinanciado con fondos FEDER). Los autores agradecen a Beatriz Panero su colaboración en la elaboración de la base de datos.

\section{Referencias}

- Calvo, A.I., 2009: Caracterización y transporte del aerosol atmosférico: medio urbano, rural y quema de biomasa. Tesis doctoral. Universidad de León. 
- NOAA (2017). Air resources laboratory. Disponible en: https://www.ready.noaa.gov/READYamet. php (Accedido 9 de octubre de 2017).

- Núñez, S. 2002: Altura de la capa de mezcla: caracterización experimental y aplicación de un modelo meteorológico para el estudio de su evolución diurna. Tesis doctoral. Universidad Complutense de Madrid.

- Seidel, D. J., Zhang, Y., Beljaars, A., Golaz, J-C., Jacobson, A.R. y Medeiros, B. 2012: “Climatology of the planetary boundary layer over the continental United States and Europe", Journal of Geophysical Research. doi: 10.1029/2012JD018143, 2012.

- Selvaratnam, V. Ordóñez, C. y Adam, M. 2015: "Comparison of planetary boundary layer heights from Jenoptik ceilometers and the unified model", Met office.

- Zhang, Y. Seidel, D.J. y Zhang, S. 2013: “Trends in planetary boundary layer height over Europe”, Journal of Climate, (26), pp.10071-10076.

- Zou, J., Sun, J., Ding, A., Wang, M., Guo, W. y Fu, C. 2017: “Observation based estimation of aerosol-induced reduction of planetary boundary layer height", Advances in Atmospheric Sciences, (34), pp. 1057-1068. 